\title{
Wireless Ad hoc Networks: A Survey of MAC based Routing Mechanisms
}

\author{
M. Thenmozhi \\ Assistant Professor \\ Department of Information Technology \\ Faculty of Engineering \\ Avinashilingam Institute for Home Science and \\ Higher Education for Women, University \\ Coimbatore.
}

\author{
S. Sivakumari, $\mathrm{PhD}$ \\ Professor \& Head \\ Department of Computer Science and Engineering \\ Faculty of Engineering \\ Avinashilingam Institute for Home Science and \\ Higher Education for Women, University \\ Coimbatore.
}

\begin{abstract}
With the expansion of ubiquitous computing stages and handheld wireless devices, wireless ad hoc networks had ended up pushed inquire about region in the field of wireless communication so as to give information communications among devices in spite of their physical areas. Media get to control without further ado called as MAC assumes an indispensable part in wireless communication stack and it has gotten requesting research consideration in the field of wireless ad hoc networks.

Wireless ad hoc networks are particularly described by the few variables which recognize them from traditional PC networks and reliable routing is the constantly requesting errand in such networks. As of late, to drop down the radio clog and for acquiring better throughput, writings on MAC have been contemplated. In this overview explore work MAC based routing systems for enhancing dependability in wireless ad hoc networks has been inspected.
\end{abstract}

From the survey certain discoveries for seeking after future research work are gotten.

\section{Keywords}

Wireless ad hoc networks, MAC, wireless communications, throughput, reliability.

\section{INTRODUCTION}

Wireless ad hoc network is a sort of wireless networks, made out of the group of mobile devices and access points (APs) in which successful communication requests setting up energy efficient, high throughput, and low latency. The radio interface is the essential energy consumption criteria in which devices deplete the accessible battery limit. Transmit, receive, idle, and sleep are the accessible radio interface modes which has maximum power consumption in the transmit mode and minimum power consumption in the sleep mode. To the extent layered design is concerned, the medium access control (MAC) sub-layer settles the mobile nodes to share the transmission medium in a wireless network alongside controlling the operations of their radio interfaces.

Thus, MAC assumes a huge part in accomplishing high throughput, low latency, and energy proficiency in wireless networks. Macintosh protocols relating to wireless networks are named contention-free and contention-based schemes. The contention-free plan makes utilization of pre-characterized assignments keeping in mind the end goal to allow nodes to transmit without contention. It likewise incorporates timedivision, polling, and token-based MAC protocols. In contention-based MAC, a hub powerfully fights with different nodes to access the channel. Despite the fact that amid the information movement load is high, there is a likelihood of collisions amid packet transmission. The radio data transfer capacity and the energy for transmitting and accepting a packet are squandered amid the transmission collision happen.

Hence, an efficient MAC plan is to be proposed which is sufficiently able to minimize the odds of transmission collisions keeping in mind the end goal to decrease energy and channel time wastage in a wireless network.

\section{RELATED WORKS}

F. Wang et al [2009] have proposed a game-theoretic MAC design named GMAC that does not depend on any connections in the offered traffic. It settles on power-control choices on a for every packet premise. Instead of settling on power choices in parallel, as in past work, every recipient utilizes its information of beforehand scheduled transmissions in its region to choose whether to join this schedule or not. GMAC bolsters a CSMA/CA-like access system for conveying TPs to fighting transmitters, henceforth empowering more simultaneous transmissions than in already proposed strategies. The protocol is offbeat, totally distributed, and utilizes just a solitary channel for both data and control packets. This guarantees equipment similarity with the 802.11 plan. The authors additionally acquainted a straight evaluating capacity with acquire Pareto enhancements in the accomplished NE arrangement.

The research issue of developing a power-aware medium access control (MAC) algorithm for Ad hoc wireless networks is proposed by A. Ghasemi and K. Faez [2010]. Depending on the insights the authors examined the optimization framework as a random scheduling MAC. By defining a payoff for each link as a function of its reliable probability and power, the objective of their proposed non-cooperative static poweraware MAC game (PAMG) is to recognize the relevant strategy for the link. Their game theoretic aspects of PAMG including existence, uniqueness, and convergence to the equilibrium are examined in an analytical manner through certain scenarios. During every non-active state in the time slot the channel observes to capture the other active links messages and frequently updates the corresponding cost factor. Their Simulation results were evaluated for the convergence and performance of the algorithm and are compared to the other optimal solutions.

D.J. Deng [2011] formed the priority enforced slow-start backoff algorithm (PSSB) for multimedia transmission for wireless ad-hoc networks. PSSB made use of a distributed adaptive contention window control mechanism to mitigate intensive collisions in congested scenario and support priority traffic for multimedia transmission. In addition to that, their 
proposed scheme could discriminate the fairness problem over the less connected network topology by making use of feedback information from wireless nodes in the network. Their simulations evaluated the performance of their proposed scheme and the author claimed that their proposed scheme outperforms the BEB algorithm in many aspects for wireless ad hoc networks.

A. Swaminathan et al. [2012] proposed a packet scheduler and a medium access control (MAC) protocol are for a directsequence spread-spectrum wireless ad hoc network. It contained a mix of nodes with directional antennas and nodes with omnidirectional antennas. The scheduler and MAC protocol are designed for avoiding the co-site interference problem that arises in certain types of ad-hoc nodes that made use of directional antennas. Their research work portrayed that the presence of nodes with directional antennas opvercomes the vulnerability of the network to the receiver blocking problem. The authors claimed that certain level of improvements with the performance of a network that includes nodes with directional antennas.

M. Khalida et al. [2013] proposed a fully distributed DMAC protocol that cooperatively makes use of polarization diversity in low-mobility urban/suburban outdoor wireless ad hoc network environment. In their proposed cooperative polarization DMAC protocol (CPDMAC), each node in the network directionally senses on both vertical and horizontal polarizations and dynamically adapts polarization that minimizes overall interference. Analysis are carried out by the authors for establishing relationship between vertically and horizontally polarized nodes in the network. Alsor, a theoretical lower bound is derived for probability of successful transmission to project the capacity improvement as a function of cross polarization ratio (CPR). Their simulation results affirmed that from $2 \%$ up to $400 \%$ improvement in average node throughput at data rate of 1.95 Mbps when compared to the traditional DMAC protocol. In addition their study clearly showed that the average throughput difference increases with increasing node density when compared to the traditional DMAC protocol.

A. Antonopoulos and C. Verikoukis [2014] presented multiplayer game theoretic channel access procedures for remote data spread plans, where multiple source hubs have clashing interests. The authors proposed non-cooperative strategic game formulations, where every player distinguishes a steady state condition (NE) keeping in mind the end goal to adjust a tradeoff between the energy sparing and the data spread consummation. The commitment of their work is twofold: i) a distributed approach for foundation less ad-hoc networks where the remote hubs independently assess the NE transmission probabilities as indicated by energy-based utility capacities, and a planned approach for framework networks, where the hubs likewise act exclusively to accomplish the NE, while a focal controller is every so often used to encourage the dispersal strategy. ii) The authors additionally exhibited an explanatory probabilistic system to assess the network performance as far as energy effectiveness and finish time in the NE state.

In K. R. Malekshan et al. [2014] proposed a novel MAC conspire for a fully connected wireless network. Utilizing a transitory coordinator node, their proposed plot diminishes the energy consumption by scheduling the dynamic and rest times of node radio interfaces distributedly, and diminishes MAC overhead and transmission collisions among nodes. A node battles just once to transmit a bunch of packets, after that it will be allocated conflict spare time for transmission by the impermanent coordinator node, the length of it has packets ready for transmission. Nodes remain alert for a brief span toward the start of every beacon interval (to get the transmission scheduling data) and amid their packet transmission/gathering times. The MAC plot means to fulfill delay and packet loss rate necessities and lessens energy consumption of nodes with ongoing movement, for example, voice or video calls that have stringent delay and packet loss prerequisites.

W. Na et al. [2015] proposed directional MAC protocol for wireless ad hoc networks that is alluded to as deafness-aware MAC (DA-MAC). Their proposed DA-MAC protocol is sufficiently proficient to recognize the deafness problem from collisions by utilizing coherent information and control channels. The authors likewise gave a discrete-time Markov bind model to dissect the effect of deafness for both a current method and DA-MAC. Through broad reproductions, they demonstrated that their DA-MAC protocol can essentially beat the other existing systems regarding the throughput, deafness duration, energy consumption, and transmission fairness.

N. C. Wang and Y. C. Huang [2015] proposed SDMA-based MAC protocol (S-MAC) for wireless ad hoc networks with savvy recieving wires. Their proposed protocol misuses the SDMA framework to permit gathering of more than one packet from spatially separated transmitters. Utilizing SDMA innovation gives impact free access to the correspondence medium in view of the area of a node. Their proposed protocol takes care of the hidden terminal problem, the uncovered terminal problem, and the deafness problem. Their reenactment comes about demonstrated the adequacy of the proposed S-MAC in enhancing throughput and expanding spatial channel reuse.

S. S. Wang et al. [2015] exhibited an aloof self-configuration MAC protocol, called PSC-MAC, to bolster arranges administration in IEEE 802.11-based MH-MANETs. The primary target of PSC-MAC is to decide appropriate STAs, called supervisors, to transmit beacon frames from the perspective of the MAC sublayer rather than the sensible connection control sublayer. PSC-MAC utilizes a beacon interleaving procedure to hinder the neighboring supervisors to transmit beacon frames in a similar beacon interim. In PSCMAC, time is made out of back to back rehashed periods, called beacon interleaving cycles(BICs). To lessen the measure of control overhead, each STA in PSC-MAC usesa detached way to figure out if it must turn into a boss in a BIC (i.e., STAs just latently hear the got beacon frames to settle on the choice). The decided supervisors shape a virtual backbone, and they are isolated into various managing sets. The STAs in a particular regulating set must wake up to transmit beacon frames in a similar beacon interims of BICs. In a beacon interim, the STAs which transmit beacon frames are known as the active supervisors, and non-active supervisors are known as the torpid supervisors.

M. Bakhouya et al. [2015] have proposed an algorithm which prompts to the broadcast storm issue that extremely influences the energy utilization because of repetitive entries. To manage excess entries, which can bring about more collisions and requires more energy, as of late there have been created various broadcasting systems. These systems have been for the most part proposed to take care of the storm issue by keeping certain nodes from rebroadcasting got messages as well as by separating the planning of rebroadcasts. In this paper, the creators have likewise assessed and looked at versatile data dispersal (AID) algorithm with different 
MANETs broadcasting protocols regarding the energy effectiveness. In AID, every node can powerfully alter the estimations of its nearby parameters utilizing data from neighboring nodes without requiring any extra exertion, for example, distance estimations or correct location assurance of nodes.

M. Sami et al. [2015] have proposed a novel cross-layer Energy-Aware PNC-based Cooperative MAC protocol, in particular EAP-CMAC. In addition, a joint relay selection and power allocation scheme is composed, which enhances the system lifetime. The proposed relay selection considers vitality utilization and the best relay is chosen based on lingering vitality and the versatile nodes' position. EAPCMAC, a good protocol, chooses the most appropriate relay node and transmission strategy among direct, cooperative and PNC-based transmissions as per the destination queue and source-destination link quality.

K. Liu et al. [2015] proposed a cooperative MAC protocol with rapid relay selection (RRS-CMAC) to enhance the cooperation efficiency and multiple access execution in wireless ad hoc networks. In this protocol, if the data rate between a sender and its recipient is low, an optimal relay is chosen by a rate differentiation phase (RDP), priority differentiation phase (PDP), and contention resolution phase (CRP) for relays with a similar priority. In the RDP, each fighting relay decides its data rate level based on the data rate from the sender to itself and that from itself to the recipient, and after that broadcasts occupied tones to its neighbor nodes or faculties the channel as indicated by the estimations of its parallel digits, which are dictated by its data rate level. Relays with the most astounding data rate levels win and proceed to the following phase. In PDP, these triumphant relays send occupied tones or sense the channel as indicated by their own particular priority values, with the most noteworthy priority relays winning in this phase. At that point CRP is performed utilizing k-round contention resolution ( $\mathrm{k}-\mathrm{CR}$ ) to choose a remarkable optimal relay. Relays sending occupied tones most punctual and for the longest length continue to the following round, while others, detecting a bustling tone, in this manner pull back from contention. A packet piggyback component is adopted to permit data packet transmission without reservation if the triumphant relay has a packet to send, and the direct transmission rate to its recipient is high. This decreases reservation overhead and enhances channel utilization.

X. Wang and J. Li [2015] exhibited a novel cross-layer distributed energy-adaptive location-based CMAC protocol DEL-CMAC that spotlights on the network lifetime augmentation, which is a less investigated perspectives in the related work. By considering the overheads and obstruction because of participation, and also the energy utilization on both handset hardware and transmit enhancer, DEL-CMAC can altogether draw out the network lifetime. A distributed energy-mindful location-based best hand-off determination technique is joined, which is more sensible for MANETs contrasting and the current plans based on channel condition. For a sought blackout likelihood necessity, a cross-layer ideal transmitting power allocation plan is designed to save the energy while keeping up certain throughput level. To manage the nearness of hand-off terminals and element transmitting power, the authors gave an imaginative NAV setting to keep away from the crashes and upgrade the spatial reuse.

K. R. Malekshan et al. [2016] proposed another medium access control (MAC) component to upgrade range effectiveness and diminish energy utilization in a remote ad hoc network. An arrangement of facilitator hubs, distributed in the network territory, intermittently schedule dispute leisure vacancies for all data transmissions/gatherings in the network, based on transmission demands from source hubs. Adjacent facilitators trade booking data to viably increment spatial range reuse and evade transmission crashes. Additionally, their proposed MAC conspire permits a hub to put its radio interface into a sleep mode when it is not transmitting/accepting a packet, keeping in mind the end goal to diminish energy utilization.

D. N. M. Dang et al. [2016] proposed a Multi-channel MAC protocol with Directional Antennas (MMAC-DA) that adopts IEEE 802.11 Power Saving Mechanism (PSM) and abuses various channel assets and directional recieving wires. Nodes trade control packets amid the Announcement Traffic Indication Message (ATIM) window to choose information channels and decide the bar headings which are utilized to trade information packets amid the information window. Their reenactment comes about demonstrated that MMAC-DA can enhance the network execution as far as total throughput, packet delivery ratio, energy efficiency and fairness index.

H. Zhao et al. [2016] proposed E-MAC that utilizations expand, rather than irregular, backoff mechanism to iteratively accomplish collision-free get to. The fundamental thought of E-MAC lies in that, each node transmits at most once in a given time cycle, and on the off chance that one node encounters a collision, it will change its transmission time in the following cycle as indicated by which some portion of its packets experiencing the collision. In particular, if the front part of the parcel is impacted, it will transmit last in the following cycle; then again, if the back part is impacted, it will transmit prior in the following cycle. The creators additionally altered the 802.11 MAC to actualize this thought and demonstrated the viability of the change by means of both hypothetical examination and $\mathrm{PC}$ reenactment.

\section{FINDINGS AND CONCLUSIONS}

A few research works have been checked on in this survey research article, for example, back - off mechanism, passive self-configuration, broadcasting techniques, adaptive information dissemination, cross-layer design, rapid relay selection, game-theoretic design, multi-player game theoretic channel access strategies, location-based, contention-free time slots data transmissions, discrete-time Markov chain model, power saving mechanism. From this survey it has been found that there exists parcel of extension for further research work which incorporates scheduling concurrent transmission, multiple route determination and bandwidth based multipath routing that can astoundingly improve the throughput and packet delivery ratio in wireless ad hoc networks.

\section{REFERENCES}

[1] F. Wang, O. Younis, M. Krunz, "Throughput-oriented MAC for mobile ad hoc networks: A game-theoretic approach," Ad Hoc Networks, vol. 7, pp. 98 - 117, 2009.

[2] A. Ghasemi, K. Faez, "A non-cooperative game approach for power-aware MAC in ad hoc wireless networks," Computer Communications, vol.33, no.12, pp.14401451,2010

[3] D.J. Deng, "PSSB: Priority enforced slow-start backoff algorithm for multimedia transmission in wireless ad-hoc networks," Journal of Network and Computer Applications, vol.34, no.5, pp.1468-1473, 2011. 
[4] A. Swaminathan, D. L. Noneaker, Harlan B. Russell, "The design of a channel-access protocol for a wireless ad hoc network with sectored directional antennas," Ad Hoc Networks, vol.10, no.3, pp.284-298, 2012.

[5] M. Khalida, X. H. Le, I. Ra, R. Sankar, "Polarizationbased cooperative directional MAC protocol for ad hoc networks," Ad Hoc Networks, vol.11, no.4, 2013.

[6] A. Antonopoulos, C. Verikoukis, "Multi-Player Game Theoretic MAC Strategies for Energy Efficient Data Dissemination," IEEE Transactions on Wireless Communications, vol. 13, pp. 592-603, 2014.

[7] K. R. Malekshan, W. Zhuang, Y. Lostanlen, "An Energy Efficient MAC Protocol for Fully Connected Wireless Ad Hoc Networks," IEEE Transactions on Wireless Communications, vol. 13, pp. 5729-5740, 2014.

[8] W. Na, L. Park, S. Cho, "Deafness-aware MAC protocol for directional antennas in wireless ad hoc networks," Ad Hoc Networks, vol. 24, pp. 121 - 134, 2015.

[9] N. C. Wang, Y. C. Huang, "An SDMA-based MAC protocol for wireless ad hoc networks with smart antennas," Computers \& Electrical Engineering, vol. 41, pp. $383-394,2015$.

[10] S. S. Wang, C. C. Li, H. W. Lin, K. P. Shih, "A passive self-configuration MAC protocol for supporting network management in IEEE 802.11-based multi-hop mobile ad hoc networks," Journal of Network and Computer Applications, vol. 56, pp. 149 - 157, 2015.

[11] M. Bakhouya, J. Gaberb, P. Lorenz, "Energy evaluation of AID protocol in Mobile Ad Hoc Networks," Journal of
Network and Computer Applications, vol. 58, pp. 287 293, 2015.

[12] M. Sami, N. K. Noordin, F. Hashim, S. Subramaniam , A. A. Moghanjoughi, "An Energy-Aware Cross-Layer Cooperative MAC Protocol for Wireless Ad Hoc Networks," Journal of Network and Computer Applications, vol. 58, pp. 227 - 240, 2015.

[13] K. Liu, X. Chang, F. Liu, X. Wang, A. V. Vasilakos, "A cooperative MAC protocol with rapid relay selection for wireless ad hoc networks," Computer Networks, vol. 91, pp. $262-282,2015$.

[14] X. Wang, J. Li, "Improving the Network Lifetime of MANETs through Cooperative MAC Protocol Design," IEEE Transactions on Parallel and Distributed Systems, vol. 26, pp. 1010-1020, 2015.

[15] K. R. Malekshan, W. Zhuang, Y. Lostanlen, "Coordination-based Medium Access Control With Space-reservation for Wireless Ad Hoc Networks," IEEE Transations on Wireless Communications, Vol. 15, 2016

[16] D. N. M. Dang, V. Nguyen, H. T. Leb, C. S. Hong, J. Choe, "An efficient multi-channel MAC protocol for wireless ad hoc networks," Ad Hoc Networks, vol. 44, pp. 46 - 57, 2016.

[17] H. Zhao, J. Wei, N. I. Sarkar, , S. Huang, "E-MAC: An evolutionary solution for collision avoidance in wireless ad hoc networks," Journal of Network and Computer Applications, vol. 65, pp. 1-11, 2016. 